\title{
Expression and regulation of macrophage- inducible C-type lectin in human synovial macrophages
}

\author{
MITSUTOSHI MORIYA ${ }^{l}$, KENTARO UCHIDA ${ }^{1,2}$, SHOTARO TAKANO ${ }^{l}$, DAI IWASE ${ }^{l}$, GEN INOUE ${ }^{l}$, \\ MANABU MUAKI', RYO TAZAWA ${ }^{l}$, JUN AIKAWA ${ }^{l}$, HIROYUKI SEKIGUCHI ${ }^{2}$, MASASHI TAKASO ${ }^{l}$ \\ ${ }^{1}$ Department of Orthopedic Surgery, Kitasato University School of Medicine, Sagamihara City, Kanagawa, Japan \\ ${ }^{2}$ Shonan University of Medical Sciences Research Institute, Chigasaki City, Kanagawa, Japan
}

\begin{abstract}
Recent evidence suggests that synovial macrophage activation may be involved in cartilage destruction and pain in osteoarthritis (OA). The macrophage-inducible C-type lectin (Mincle) Clec4e is expressed in macrophages and is regulated in inflammatory conditions. Given that the regulation of Mincle in synovial macrophages has not been elucidated, we investigated the expression and regulation of Mincle in human synovial tissue (ST) harvested from patients with radiographic knee OA during total knee arthroplasty. Immunohistochemical and flow cytometric analyses were used to identify cells with Mincle expression in resected tissues. CD14-positive $\left(\mathrm{CD}^{+} 4^{+}\right.$; macrophage-rich cell fraction) and CD14-negative (CD14-; fibroblast-rich cell fraction) cells were extracted from the ST and used to assess MINCLE mRNA expression levels. To determine the role of tumor necrosis factor alpha $(T N F-\alpha)$ in the regulation of MINCLE expression, TNF- $\alpha$ was used to stimulate cultured CD14+ cells. Immunohistochemical staining revealed Mincle-positive cells in the synovial lining layer. Flow cytometric analysis showed that $\mathrm{CD} 45^{+} \mathrm{CD} 14^{+}$cells were Mincle positive while CD45-/CD14- cells were Mincle negative. MINCLE expression was significantly higher in CD14+ cells than in CD14- cells. Stimulation of cultured CD14+ macrophages with TNF- $\alpha$ significantly increased MINCLE mRNA expression, while stimulation with TNF- $\alpha$ neutralizing antibody significantly decreased expression. That Mincle expression was observed in synovial macrophages and its expression was induced by TNF- $\alpha$ suggests that Mincle might have a key role in synovial inflammation in the osteoarthritic synovium.
\end{abstract}

Key words: osteoarthritis, synovial tissue, Mincle, tumor necrosis factor- $\alpha$.

(Cent Eur J Immunol 2020; 45 (4): 377-381)

\section{Introduction}

Knee osteoarthritis (KOA), a degenerative joint disease, is commonly typified by articular cartilage degeneration, osteophytosis, and bone remodeling in addition to fibrosis and synovial hyperplasia [1]. Additionally, increasing evidence indicates that synovial inflammation is involved in a number of the signs and symptoms underlying KOA, such as joint effusion and swelling. Synovial inflammation in KOA is likely to contribute to disease progression along with the progression of structural changes in KOA [2]. While synovium inflammation is a key player in KOA pathology, the molecular signals responsible for the regulation of synovial inflammation have not been fully determined.

The macrophage-inducible C-type lectin (Mincle) Clec4e is a transmembrane pattern recognition receptor with roles in innate immunity [3, 4]. Mincle is predominantly expressed in monocytes/macrophages and is stimulated in several inflammatory conditions [5-11]. Human blood monocytes and bone marrow mononuclear cells (BMMCs) obtained from rheumatoid arthritis (RA) patients showed higher expression of MINCLE than the healthy control sample $[10,11]$. Previous studies have shown that there are high levels of Mincle in macrophages in adipose tissue from obese humans and mice [6] and in a cisplatin-induced renal injury murine model [7].

The synovial tissue (ST) is a highly specialized structure with multiple functions. It comprises a thin but highly cellular lining layer of synovial fibroblasts and synovial macrophages [12], in addition to a second layer. Macrophages are one of the major sources of inflammatory signals in the ST of KOA patients and may therefore contribute to joint destruction and pain [13-15]. However, the expression of Mincle has not been examined in synovial macrophages from KOA patients.

Correspondence: Kentaro Uchida, Department of Orthopedic Surgery, Kitasato University School of Medicine, 1-15-1 Minami-ku Kitasato, Sagamihara City, Kanagawa 252-0374, Japan, e-mail: kuchida@med.kitasato-u.ac.jp Submitted: 30.08.2018, Accepted: 25.10.2018 
A number of recent studies suggest that tumor necrosis factor alpha (TNF- $\alpha$ ) levels are increased in ST, and that this inflammatory signal is associated with KOA pathology $[13,14,16,17]$. Synovial macrophages, in particular M1 macrophages (classically activated macrophages), produce TNF- $\alpha$ and induce the expression of matrix metalloprotases and a disintegrin and metalloproteinase with thrombospondin motifs 4 (ADAMTS4) in synovial fibroblasts extracted from a KOA mouse model and KOA patients $[14,15]$. Moreover, several inflammatory stimuli have been shown to induce high levels of Mincle in macrophages [5, 8, 9] to contribute to maintaining the inflammatory state [8]. Based on these findings, we speculated that TNF- $\alpha$ may regulate Mincle expression in synovial macrophages.

Here, we investigated Mincle expression and regulation in the ST of KOA patients.

\section{Material and methods}

\section{Patients}

This study included 19 subjects with radiographic KOA (unilateral Kellgren/Lawrence [K/L] grades 3-4) who received total knee arthroplasty at our institution. Among them, there were 4 men and 15 women aged 56-85 years (mean \pm standard deviation [SD], $72.2 \pm 7.0$ years) with a mean \pm SD body mass index (BMI) of $25.5 \pm 3.2 \mathrm{~kg} / \mathrm{m}^{2}$ (range 18.8-30.9). An ST sample was extracted from each operated knee during surgeries. This study was approved by the Ethics Review Board of Kitasato University (reference number: KMEO B13-113). All participants gave consent one day prior to the surgery for the extraction and use of their ST.

\section{Immunohistochemistry}

To examine the distribution of Mincle, ST samples were fixed in $4 \%$ paraformaldehyde (PFA) formalin in phosphate buffered saline (PBS) (Wako Pure Chemicals, Osaka, Japan) for $48 \mathrm{~h}$. PFA-fixed ST samples were embedded in paraffin and sectioned at $4 \mu \mathrm{m}$ thickness before being deparaffinized in a xylene replacement solvent (Clear-plus ${ }^{\circledR}$, Pharma, Tokyo, Japan) for 1 hour. The sections were hydrated in a series of decreasing concentrations of ethanol $(100 \%, 95 \%, 80 \%$, and $75 \%)$ and subsequently rinsed in distilled water. The sections were incubated with a mouse anti-Mincle monoclonal primary antibody ( 1 : 200; clone AT16E3, GeneTex, LA, USA) and mouse anti-TNF- $\alpha$ monoclonal primary antibody $(1: 100$; clone $2 \mathrm{C} 8$, Abcam, Cambridge, UK) for 6 hours at $4^{\circ} \mathrm{C}$.

\section{Flow cytometric analysis}

Flow cytometric analysis was performed to identify the ST cells expressing Mincle [13]. ST were digested with $\alpha$-minimum essential medium ( $\alpha$-MEM, Invitrogen, CA, USA) containing $1 \mathrm{mg} / \mathrm{ml}$ collagenase solution (Sigma, MO, USA) on shaker for 2 hours at $37^{\circ} \mathrm{C}$. Extracted cells were incubated with phycoerythrin (PE)-conjugated mouse anti-CD14 monoclonal antibody ( 1 : 20; clone M5E2, Biolegend, CA, USA), fluorescein isothiocyanate (FITC)-conjugated mouse anti-CD45 monoclonal antibody (1:20; clone LCA, BD Biosciences, San Jose, CA, USA), allophycocyanin (APC)/ Cy7-conjugated anti-human CD31 (1: 20; clone WM59, Biolegend), APC-conjugated mouse anti-CD90 monoclonal antibody (1:20; clone 5E10, Biolegend), and rat anti-Mincle monoclonal antibody $(1: 200$; clone $1 \mathrm{H} 2$, MBL, Nagoya, Japan) for 45 minutes at $4^{\circ} \mathrm{C}$. The cells were washed twice in PBS, resuspended in ice cold PBS and reacted with brilliant violet 421-conjugated goat anti-rat IgG polyclonal antibody (1: 100; clone Polu4054, Biolegend). After washing twice in PBS, the cells were analyzed by flow cytometric device (FACSVerse ${ }^{\mathrm{TM}}$; BD Biosciences). Isotype controls were used as the positive gate.

\section{Isolation of CD14-positive (CD14+) and CD14-negative (CD14-) cells}

To examine the role of TNF- $\alpha$ in regulating Mincle expression, synovial fibroblasts and macrophages were extracted from the ST from the knees of 10 KOA patients. Following collagenase digestion of ST, the extracted cells were incubated with biotin-conjugated mouse anti-human CD14 monoclonal antibody (1 : 20; clone M5E2, Biolegend) for 30 minutes at $4^{\circ} \mathrm{C}$. The cells were subsequently washed twice with PBS, combined with streptavidin-conjugated magnetic particles (BD Biosciences, CA, USA), and separated in a magnetic separation system (BD IMag ${ }^{\mathrm{TM}}$ cell separation system, BD Biosciences) into $\mathrm{CD} 14^{+}$and CD14- cells, as described elsewhere [13]. CD14 ${ }^{+}$and CD14- cell fractions from five patients were immediately analyzed for TNF- $\alpha$, CD14, and Mincle expression using quantitative polymerase chain reaction (qPCR), while those from the remaining five patients were cultured for 7 days in six-well plates containing $\alpha$-MEM. The cells were subsequently stimulated with vehicle ( $\alpha$-MEM), $10 \mathrm{ng} / \mathrm{ml} \mathrm{hu-}$ man recombinant TNF- $\alpha$ (Biolegend), or $10 \mathrm{ng} / \mathrm{ml} \mathrm{TNF-} \alpha$ $+1 \mu \mathrm{g} / \mathrm{ml}$ anti-TNF- $\alpha$ antibody (clone Mab11, Biolegend) for 24 hours, before isolating RNA for real time (RT)-PCR analysis for Mincle expression.

\section{Statistical analysis}

Differences between CD14- and CD14+ cells were examined using the t-test. Comparisons between multiple groups were performed using one-way analysis of variance with Tukey's multiple comparisons test. For all analyses, $p<0.05$ indicated statistical significance. All statistical analyses were conducted using SPSS software (v. 25.0; SPSS, Chicago, IL, USA). 


\section{Results}

\section{Localization and expression of Mincle in ST of KOA patients}

Immunohistochemical and flow cytometric analyses of Mincle and TNF- $\alpha$ were performed to identify the cell type(s) within the synovial lining layer of the knees of KOA patients expressing Mincle (Figures 1 and 2). Both TNF- $\alpha$-positive and Mincle-positive cells were observed in the intimal lining layer (Figure 1A-D). Flow cytometric analysis showed that some $\mathrm{CD} 45^{+} \mathrm{CD} 14^{+}$macrophages were Mincle positive, while $\mathrm{CD} 45^{-} \mathrm{CD} 14^{-}$fibroblasts were Mincle negative. Quantitative PCR analysis demonstrated that the expression of MINCLE and TNFA was significantly greater in $\mathrm{CD} 14^{+}$fractions than in $\mathrm{CD} 14^{-}$fractions (Figure 3, $p=0.010$ ).

\section{Effect of TNF- $\alpha$ on MINCLE mRNA expression in synovial macrophages}

Stimulation of cultured CD14+ fractions with exogenous TNF- $\alpha$ significantly raised MINCLE mRNA expres-
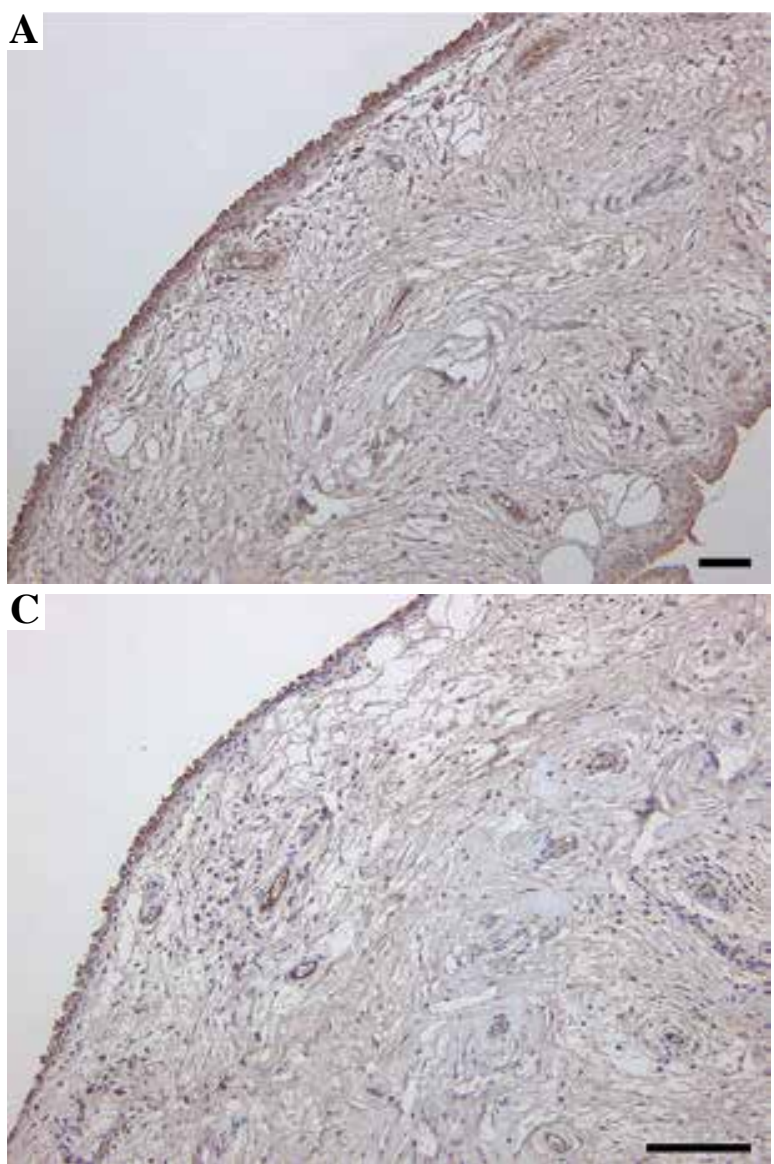

sion compared to vehicle-stimulated controls (8.9-fold, $p=0.042$ ). In contrast, stimulation with TNF- $\alpha$ neutralizing antibody significantly reduced MINCLE mRNA expression compared to exogenous TNF- $\alpha$-stimulated cells $(p=0.040)$.

\section{Discussion}

We demonstrated that Mincle-positive cells were localized to the synovial lining layer of the knees of KOA patients. $\mathrm{CD} 45^{+} \mathrm{CD} 14^{+}$cells were Mincle positive, and $\mathrm{CD}_{14}{ }^{+}$synovial cells and isolated $\mathrm{CD} 14^{+}$cells expressed high levels of MINCLE compared to CD14- cells. Moreover, stimulation of $\mathrm{CD} 14^{+}$cells with TFN- $\alpha$ induced Mincle expression. Taken together, these results suggest that Mincle may play an important role in synovial inflammation mediated by synovial macrophages.

Mincle was initially recognized as a downstream target of nuclear factor interleukin-6 (NF-IL-6; also called $\mathrm{C} / \mathrm{EBPb}$ ) in peritoneal macrophages in mice [9]. Human Mincle is expressed by myeloid cells such as circulating monocyte-derived macrophages, macrophages in adipose

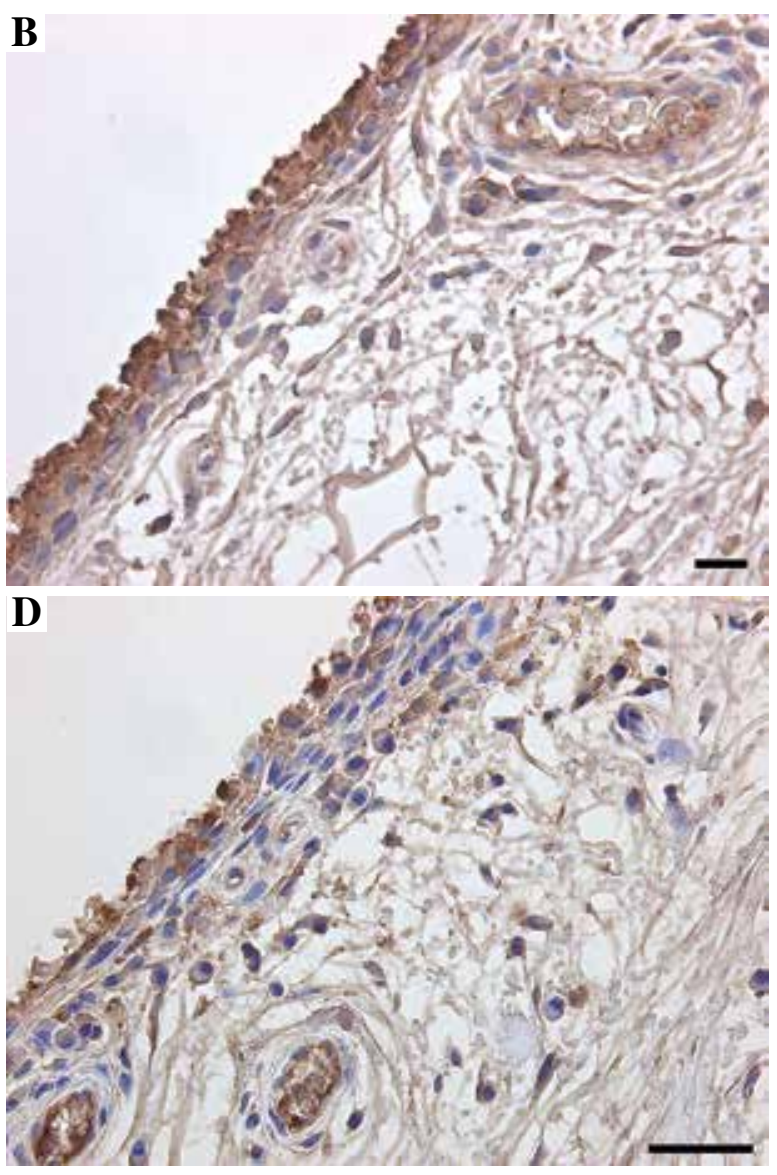

Fig. 1. Immunolocalization of Mincle and TNF- $\alpha$ in synovial tissue. Immunolocalization of Mincle (A, B) and TNF- $\alpha$ $(\mathbf{C}, \mathbf{D})$. Scale bars $=100 \mu \mathrm{m}$ 

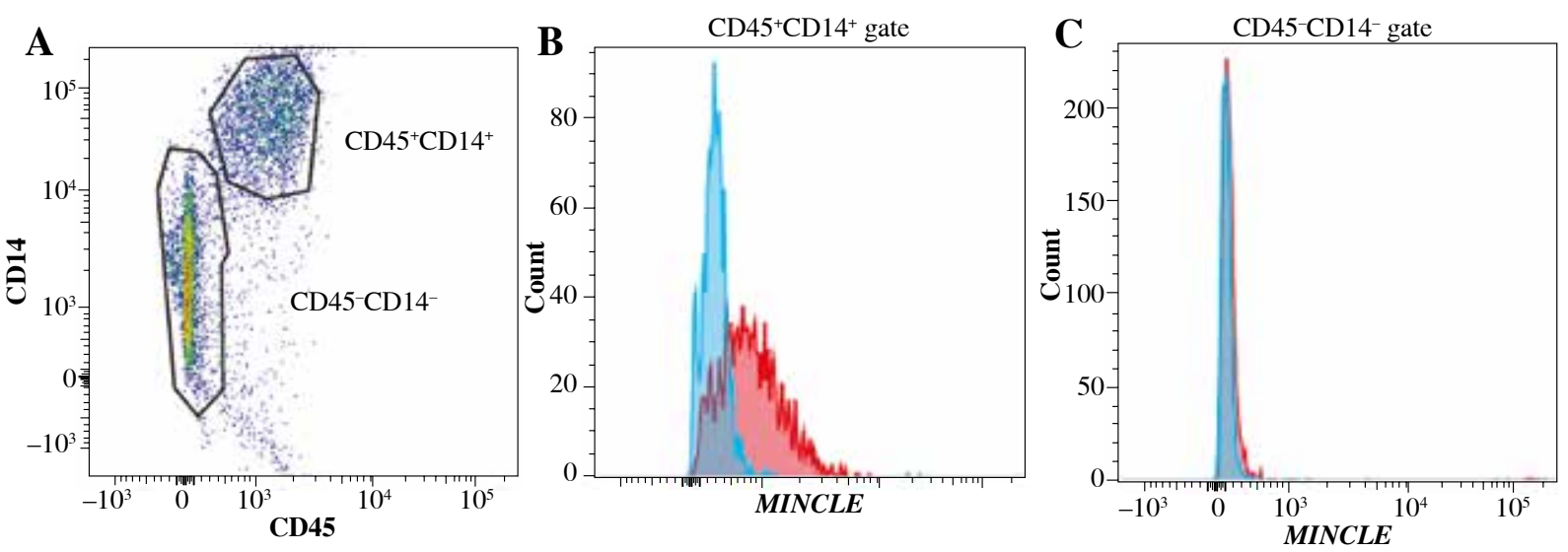

$\square$ Isotype control $\square$ Anti-Mincle antibody

Fig. 2. Flow cytometric analysis of MINCLE expression in CD14-negative and -positive synovial cells extracted from knee osteoarthritis patients. A) Dot-plot analysis of CD45+CD14+ cells and CD45-CD14- cells. X-axis, CD45; y-axis, CD14. $\mathbf{B}, \mathbf{C})$ Histogram analysis of MINCLE expression in the $\mathrm{CD} 45^{+} \mathrm{CD} 14^{+}(\mathrm{B})$ and $\mathrm{CD} 45^{-} \mathrm{CD} 14^{-}(\mathrm{C})$ gated regions in $(\mathbf{A})$
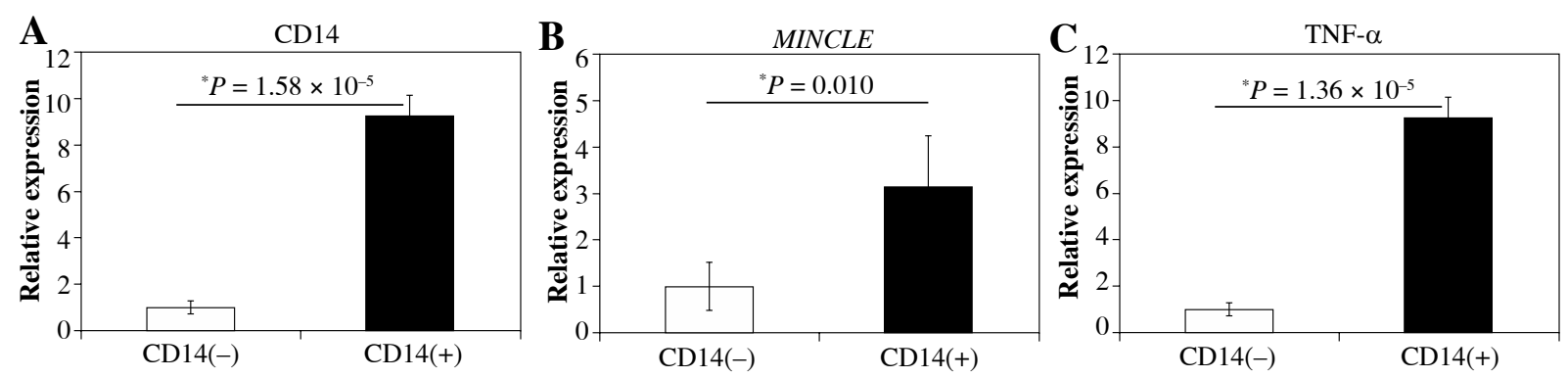

Fig. 3. Quantitative polymerase chain reaction (qPCR) analysis of CD14, MINCLE, and TNF- $\alpha$ mRNA levels in CD14-negative and -positive synovial cells extracted from knee osteoarthritis patients. A) CD14, B) MINCLE, C) TNFA. Data indicate mean \pm standard error $(\mathrm{SE})(n=8)$. *Statistical difference between CD14-negative- and -positive cells

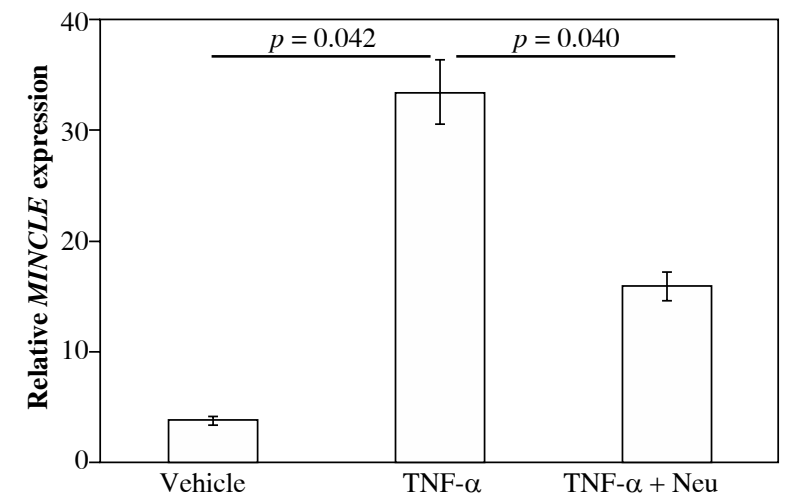

Fig. 4. Impact of tumor necrosis factor (TNF)- $\alpha$ on MINCLE expression. CD14-positive synovial cells were stimulated with vehicle $(\alpha$-minimum essential culture medium $(\alpha-M E M)], 10 \mathrm{ng} / \mathrm{ml} \mathrm{TNF-} \alpha$ or $10 \mathrm{ng} / \mathrm{ml} \mathrm{TNF-} \alpha+1 \mu \mathrm{g} / \mathrm{ml}$ TNF- $\alpha$ neutralizing antibody (TNF- $\alpha+\mathrm{Neu}$ ) for $24 \mathrm{~h}$ before the extraction and analysis of total RNA. Differences between control and TNF- $\alpha$ and between TNF- $\alpha$ and TNF- $\alpha+$ Neu groups were assessed using the Tukey's multiple comparisons test with one-way analysis of variance tissue and BMMCs $[6,10,11]$. Ichioka et al. reported that MINCLE was expressed at higher levels in BMMCs from RA than OA patients [10]. Here, we observed Minclepositive cells in the synovial lining layer of the knees of KOA patients. CD14+ synovial cells expressed higher levels of MINCLE compared to CD14- cells. A Mincle agonist injection induced skin inflammation in human Mincle $\mathrm{Tg}$ mice. Further, a Mincle agonist induced the production of TNF- $\alpha$ in human blood monocyte-derived macrophages, which was completely suppressed by an anti-human Mincle antibody [11]. These findings suggest that Mincle may have a key role in macrophage-related synovial inflammation in KOA.

Mincle is strongly induced by a number of inflammatory stimulation, such as IL-6, lipopolysaccharides (LPS), and interferon (IFN)- $\gamma$ in macrophages $[5,8,9]$. Treatment with these inflammatory mediators markedly reduced MINCLE mRNA induction in macrophages from NF-IL-6deficient mice [9]. Additionally, LPS stimulates MINCLE mRNA expression in the murine macrophage cell line RAW264.7 [8]. In the present study, Mincle and TNF- $\alpha$ were localized to the synovial lining layer and stimula- 
tion of cultured CD14+ fractions with exogenous TNF- $\alpha$ significantly raised MINCLE mRNA expression compared to vehicle-stimulated cells. Mincle activation leads to the production of chemokines and cytokines, neutrophil recruitment and other inflammatory responses [18]. Mincle signaling promotes inflammation and also maintains the inflammatory state in acute renal inflammation in mice [8]. TNF- $\alpha$-mediated MINCLE expression may be a key component in synovial inflammation.

In conclusion, Mincle expression was observed in synovial macrophages and was induced by the presence of TNF- $\alpha$. Mincle may have a key role in maintaining synovial inflammation in the osteoarthritic synovium.

\section{Acknowledgements}

This investigation was supported in part by a research grants from the Parents' Association of Kitasato University School of Medicine., JSPS KAKENHI Grant No. 18K09119, Grant of Japan Orthopaedics and Traumatology Research Foundation, Inc. No. 373, and JSPS KAKENHI Grant No. 18K09119.

The authors declare no conflict of interest.

\section{References}

1. Lietman C, Wu B, Lechner S, et al. (2018): Inhibition of Wnt/ beta-catenin signaling ameliorates osteoarthritis in a murine model of experimental osteoarthritis. JCI Insight 3: e96308.

2. Bondeson J, Wainwright SD, Lauder S, et al. (2006): The role of synovial macrophages and macrophage-produced cytokines in driving aggrecanases, matrix metalloproteinases, and other destructive and inflammatory responses in osteoarthritis. Arthritis Res Ther 8: R187.

3. Richardson MB, Williams SJ (2014): MCL and Mincle: C-type lectin receptors that sense damaged self and pathogen-associated molecular patterns. Front Immunol 5: 288.

4. Yamasaki S, Ishikawa E, Sakuma M, et al. (2008): Mincle is an ITAM-coupled activating receptor that senses damaged cells. Nat Immunol 9: 1179-1188.

5. Behler F, Steinwede K, Balboa L, et al. (2012): Role of Mincle in alveolar macrophage-dependent innate immunity against mycobacterial infections in mice. J Immunol 189: 3121-3129.

6. Ichioka M, Suganami T, Tsuda N, et al. (2011): Increased expression of macrophage-inducible C-type lectin in adipose tissue of obese mice and humans. Diabetes 60: 819-826.

7. Inoue T (2017): M1 macrophage triggered by Mincle leads to a deterioration of acute kidney injury. Kidney Int 91: 526-529.

8. Lv LL, Tang PM, Li CJ, et al. (2017): The pattern recognition receptor, Mincle, is essential for maintaining the M1 macrophage phenotype in acute renal inflammation. Kidney Int 91: 587-602.

9. Matsumoto M, Tanaka T, Kaisho T, et al. (1999): A novel LPS-inducible C-type lectin is a transcriptional target of NFIL6 in macrophages. J Immunol 163: 5039-5048.

10. Nakamura N, Shimaoka Y, Tougan T, et al. (2006): Isolation and expression profiling of genes upregulated in bone marrow-derived mononuclear cells of rheumatoid arthritis patients. DNA Res 13: 169-183.

11. Hattori Y, Morita D, Fujiwara N, et al. (2014): Glycerol monomycolate is a novel ligand for the human, but not mouse macrophage inducible C-type lectin, Mincle. J Biol Chem 289: 15405-15412.

12. Kurowska-Stolarska M, Alivernini S (2017): Synovial tissue macrophages: friend or foe? RMD Open 3: e000527.

13. Takano S, Uchida K, Inoue G, et al. (2017): Nerve growth factor regulation and production by macrophages in osteoarthritic synovium. Clin Exp Immunol: 190: 235-243.

14.Uchida K, Satoh M, Inoue G, et al. (2015): CD11c(+) macrophages and levels of TNF-alpha and MMP-3 are increased in synovial and adipose tissues of osteoarthritic mice with hyperlipidaemia. Clin Exp Immunol 180: 551-559.

15.Uchida K, Takano S, Matsumoto T, et al. (2017): Transforming growth factor activating kinase 1 regulates extracellular matrix degrading enzymes and pain-related molecule expression following tumor necrosis factor-alpha stimulation of synovial cells: an in vitro study. BMC Musculoskelet Disord 18: 283.

16. Grunke M, Schulze-Koops H (2006): Successful treatment of inflammatory knee osteoarthritis with tumour necrosis factor blockade. Ann Rheum Dis 65: 555-556.

17. Guler-Yuksel M, Allaart CF, Watt I, et al. (2010): Treatment with TNF-alpha inhibitor infliximab might reduce hand osteoarthritis in patients with rheumatoid arthritis. Osteoarthritis Cartilage 18: 1256-1262.

18. Lu X, Nagata M, Yamasaki S (2018): Mincle: 20 years of a versatile sensor of insults. Int Immunol 30: 233-239. 